\title{
Demokratie und moralische Führerschaft. Die Funktion praktischer Kritik für den Prozess partizipativer Demokratie
}

\author{
Stefan Müller-Doohm / Klaus Neumann-Braun \\ Unter Mitarbeit von Armulf Deppermann
}

\section{Demokratie, Öffentlichkeit und Kritik}

Die apriorische Bedingung für das entwickelte Modell einer deliberativen Demokratie besteht in einer funktionsfähigen autonomen Öffentlichkeit. Die Institutionalisierung von Öffentlichkeit und die dynamisch wirksame Pluralität ihrer Stimmen durch die Partizipation verschiedener Handlungsakteure ist die zivilgesellschaftliche Quelle, aus der jene Meinungs- und Willenbildungsprozesse schöpfen, von denen nichts Geringeres erwartet wird, als den ,harten Kern (...) sozialer Macht (...) mit demokratischen Mitteln aufzulösen“ (Offe, 2003, 8). Weil es diese Interdependenz zwischen Moderne, Demokratisierung und Öffentlichkeit gibt, muss für das Niveau der öffentlichen Debatte Sorge getragen werden. Nur wenn die Öffentlichkeit mit dem Kriterium der uneingeschränkten Kommunikation zugleich dem Kriterium einer diskursiven Praxis Rechnung trägt, die der Logik des besseren Arguments im Medium von Kritik und Gegenkritik gehorcht, entfalten die öffentlichen Diskurse ihr Rationalitätspotenzial. Unter diesem Aspekt, dass sich die Qualität von Öffentlichkeit einzig durch die Impulse permanenter Kritik erhalten und steigern lässt, ist die Rolle des Intellektuellen als funktional notwendiges Element deliberativer Demokratie von Bedeutung.

Um den Funktionswandel intellektueller Praxis deutlich zu machen, ist es aufschlussreich, sich die Rolle zu vergegenwärtigen, die Theodor W. Adorno und Jürgen Habermas als öffentliche Intellektuelle jeweils in ihrer Zeit gespielt haben bzw. spielen (Müller-Doohm, 2005). Vor diesem Hintergrund lässt sich prüfen, ob der neue, tendenziell dominierende Typus des Medienintellektuellen geeignet ist, die für den Prozess der Öffentlichkeit funktional notwendige, kritisch unabhängige Urteilskraft zu entwickeln, die aus dem Spannungsverhältnis von Autonomie und Engagement resultiert. Mit anderen Worten gilt es zu fragen: Wird beim Medienintellektuellen die Logik des besseren Arguments durch den Mechanismus der Stimmungsmache, d. h. der Diskreditierung und Skandalisierung ersetzt - oder nicht? 


\section{Funktion intellektueller Praxis}

Bekanntlich hat Karl Mannheim, auch auf sich selbst bezogen, vom sozial freischwebenden Intellektuellen gesprochen; das ist einer, der zwischen allen Stühlen sitzt. Entsprechend charakterisiert er schon in seinen "Heidelberger Briefen" von 1921 die Intellektuellen als „verstreute Menge (...) ohne Grund unter den Füssen (...). Wen man wirklich in diese Kaste eingliedern kann, lässt sich nur von Fall zu Fall entscheiden“ (Karadi, Erszébet und Lukács, 1985, 75). Richtig daran ist, dass man zum Intellektuellen weder durch Geburt oder Herkunft prädestiniert, noch die Kompetenz des Intellektuellen professionalisierbar ist. Die intellektuelle Praxis ist also keine haupt- oder spezialberufliche Tätigkeit. Sie ist eher eine dem eigenen Gewissen verpflichtete Tugend, die nicht so häufig vorkommt, und auch deshalb kulturell auffällig ist. Seit der Dreyfus-Affäre werden Gebildete als Intellektuelle bezeichnet, sofern sie das Wagnis eingehen, in der Öffentlichkeit kritisch opponierend das Wort zu ergreifen (Gilcher-Holtey, 1997, 61 ff.) ${ }^{1}$. Der potentielle Intellektuelle wird durch das praktische Engagement öffentlicher Kritik zum aktuellen Intellektuellen. Kritik heißt in diesem Fall nicht die methodisch kontrollierte Falsifikationspraxis des nach objektiver Erkenntnis strebenden Berufswissenschaftler (der an das Postulat der Werturteilsfreiheit gebunden ist), sondern Kritik meint hier die situativen, zeitlich begrenzten, aber wiederholten Interventionen im Sinne bewusst kontroverser Stellungnahmen zu praktisch-politischen Problemen des Zusammenlebens. Entsprechend richtet sich die intellektuellen Interventionen zugrunde liegende normative Kritik nicht an die Wissenschaftlergemeinschaft, sondern an eine funktionsfähige, plurale Öffentlichkeit und ist Kritik an Missständen der institutionellen Ordnung, Kritik an bestimmten fragwürdigen politischen Praktiken, ist Kritik, die neue Sichtweisen zu erschließen sucht. Der Intellektuelle spricht nicht mit der Autorität des wissenschaftlichen Experten, auch wenn er in irgendeinem Bereich Experte ist, er bedient sich einer verständlichen Bildungssprache, er erlaubt sich bei seinen Interventionen rhetorische Figuren, Dramatisierungen, Polemiken, Ironie und Generalisierungen, die eine polarisierende Wirkung bei den Rezipienten haben können.

Der Intellektuelle muss das Kunststück zuwege bringen, einerseits seine relative Ungebundenheit als Wissenschaftler, Schriftsteller oder Künstler glaubwürdig zum Ausdruck zu bringen, weil diese Autonomie Hand in Hand geht mit der Anerkennung als jemand, dessen Stimme Gehör verdient. Anderseits muss er aus dieser Freiheit heraus eindeutig und überzeugend Stellung beziehen, also sich im Feld politischer Interessengegensätze positionieren (Hillmann, 1997, 80) ${ }^{2}$. Bei dieser Art

1 Jacob Taubes stellt fest: „Das 'Manifest der lntellektuellen' von 1898 inmitten der Affäre Dreyfus war im wesentlichen von der Ecol Normal Supérieure getragen und schuf die Voraussetzungen für die 'Republik der Professoren', die in den ersten Jahrzehnten des zwanzigsten Jahrhunderts die dritte Republik repräsentiene. (...) Die Affàre Dreyfus hatte die Geister in Frankreich noch eindeutig geschieden: auf der einen Seite die Mächte des ancien régime, die Kirche und das Militär, auf der anderen die Träger der Tradition der Revolution, die Intellektuellen. (...) Die Intellektuellen in Frankreich stehen darum bis heute einheitlicher als sonst links $(. . .)^{\prime \prime}$.

2 So bilden, ,intellektuelle Autonomie und politisches Engagement die zwei komplementären strukturellen Elemente. aus denen sich die Rolle des Intellektuellen konstituiert“. Henning Hillmann (1997): 
der praktischen Kritik muss der Intellektuelle durchaus - dies gegen Mannheim gesagt - Grund unter die Füße bekommen, denn seine Kritik beinhaltet stets Werthaltungen. Diese Orientierung an Werten bzw. der Widerstand gegen ihre Missachtung ist das eigentliche Motiv dafür, die Rolle des Intellektuellen zu übernehmen. In dieser Rolle ist er nicht nur ein Interpret der gesellschaftlich anerkannten Grundwerte, vielmehr erinnert der Intellektuelle die Gesellschaft an ihre eigenen normativen Vorgaben und die Verfehlung dieser Vorgaben. Die Wertposition ergibt sich jedoch nicht aus einer per se festgelegten professionsethischen oder parteipolitischen Bindung, nicht aus der Parteilichkeit unmittelbarer Interessen. Wissenschaftler, Literaten, Journalisten oder Künstler, die öffentliche Kritik üben, werden als Intellektuelle positiv oder negativ wahrgenommen, weil ihre Position als eine weitgehend autonome oder neutrale gelten kann. Diese Form der „strukturellen Ortlosigkeit“ (Oevermann, 2001, 13 f.) ${ }^{3}$ im Dazwischen von geistiger Autonomie und politischem Engagement in der Sphäre der Öffentlichkeit hat Karl Mannheim im Auge, wenn er die Ungebundenheit des Intellektuellen betont.

\section{Adorno: Der agonale Intellektuelle}

Adorno, einer der schärfsten Kritiker von Mannheim, versucht zwar in seinen Reflexionen zur paradoxen Position des Intellektuellen, die Widersprüchlichkeit dieses sozialen Typus strukturell präziser zu bestimmen. Aber wenn er sich selbst als die eigentlich untergegangene Gestalt des Intellektuellen im Niemandsland platziert und in den Minima Moralia feststellt, die Intellektuellen seien die letzten Feinde der Bürger und zugleich die letzten Bürger, dann steht zumindest diese Sicht im Einklang mit seinen Kontrahenten aus den dreißiger Jahren (Adorno, 1951/1997, 28). Jedoch deutlicher als diese hat Adomo die Aporien der Rolle des Intellektuellen in der spätbürgerlichen, in der integralen, der vergesellschafteten Gesellschaft thematisiert. Dabei ist Adornos Blick auf den Intellektuellen durch seine über anderthalb Jahrzehnte umfassende Exilerfahrung geschärft. Sie ist ausschlaggebend für seine Politisierung sowie für seine Selbstbeschreibung als Intellektueller, dessen Weltsicht

Zwischen Engagement und Autonomie: Elemente für eine Soziologie des Intellektuellen. in: Berliner Journal für Soziologie. Bd. 7, S. 71-86, S. 80.

3 In seinem Aufsatz schlägt Oevermann eine Definition des Intellektuellen vor, die eine präzise soziologische Bestimmung des sozialen Ortes und der Funktion des Intellektuellen beinhaltet: „Ein Intellektueller ist eine Person, die kraft einer geistigen Leistung der Argumentation oder der extmplifizierenden Darstellung zu einem aktuellen, offenen Thema, das die Öffentlichkeit als Forum einer politisch-kulturellen Vergemeinschaftung insgesamt in ihrem Selbstwert zentral etwas angeht und deshalb die für sie konstitutiven Werthaltungen zentral in ihrem Bestand und ihrer Geltung herührt, in der Lage und fähig ist, sich innerhalb dieser Öffentlichkeit ad hoc eine Gefolgschaft des Gehörs zu verschaffen, wobei diese Gefolgschaft nicht durch inhaltliche Affirmation, Zustimmung oder Konformität gekennzeichnet ist, wie im Falle des Charisma des politischen Führers, sondern durch ein Hervorrufen bzw. das Fortühren einer Strittigkeit eines Pro und Contra von propositio und oppositio. Ob man Intellektueller ist oder nicht, stellt sich erst im Vollzug einer Argumentations- bzw. einer darstellerischen Exemplifizierungsleistung her, die sich erfolgreich hinreichend Gehör verschaffen kann, so dass sie eine strittige Debatte nach sich zieht oder weiter entwickelt, auf die von da an dic öffentliche Diskussion Bezug nehmen muss“" (ebd. S. 20 f.). 
durch die Marginalität des Außenseiters ihre distanzierte und militante Akzentuierung erhält. Nicht umsonst spricht Adorno von sich selbst als einem ,professionell Heimatlosen" (Adorno und Mann, 2002, 49). Die Bedingung der Möglichkeit für die Intransigenz der Kritik des Intellektuellen besteht Adomos Worten zufolge eben darin, dass „man (...) nirgends mehr zu Hause (ist), und darüber sollte freilich wiederum der, dessen Geschäft die Entmythologisierung ist, nicht allzu sehr sich beklagen" (ebd.).

Will sich der Intellektuelle in Solidarität mit der ganzen Menschheit für deren Freiheit einsetzen, kann er dies eigentlich nur von außen, in ,unverbrüchlicher Einsamkeit (...). Alles Mitmachen, alle Menschlichkeit von Umgang und Teilhabe ist bloße Maske fürs stillschweigende Akzeptieren des Unmenschlichen" (Adorno, 1951/1997, 27). Umgekehrt jedoch ist die Distanziertheit, die der Intellektuelle für sich in Anspruch nimmt. illusionär. .Der Distanzierte bleibt so verstrickt wie der Betriebsame (...). Die eigene Distanz vom Betrieb ist ein Luxus, den einzig der Betrieb abwirft" (ebd.). Angesichts dieser Ausweglosigkeit bleibt für den Intellektuellen jener moralische Rat in kleiner Münze, „den ideologischen Missbrauch der eigenen Existenz sich zu versagen" (ebd., S. 29).

Wieweit hat Adorno selbst, nicht als Person, sondern in seiner intellektuellen Praxis, diese Maxime realisiert? Diese Frage lässt sich mit hinreichender Deutlichkeit am Beispiel seines Beitrags zum Vergangenheits- und Schulddiskurs im Deutschland der Nachkriegsjahre diskutieren. Kurze Zeit nachdem er aus dem amerikanischen Exil nach Frankfurt zurückgekehrt ist, nimmt er sehenden Auges das Risiko auf sich, im Land des Henkers vom Strick zu sprechen (Müller-Doohm, 2003, 583); er provozier die literarische Öffentlichkeit mit dem 1951 publizierten Satz, es sei barbarisch, nach Auschwitz ein Gedicht zu schreiben. Als er so redet, war ihm klar, dass er sich mit diesem Diktum in einer Weise exponiert, die einen Rückzug in den Elfenbeinturm der reinen Wissenschaft kaum mehr zulassen würde. Er geht noch einen Schritt weiter, stellt sich vollends ins Rampenlicht der Öffentlichkeit, als er sich Ende der fünfziger und Anfang der sechziger Jahre mit Vorträgen zu Wort meldet wie: „Was bedeutet: Aufarbeitung der Vergangenheit?“ oder „Die Bekämpfung des Antisemitismus heute“ oder „Erziehung nach Auschwitz“. In dieser Zeitphase, in der antisemitische Reaktionen in Westdeutschland zu verzeichnen waren, bietet der Philosoph und Soziologe die Reputation seiner wissenschaftlichen Stellung auf, um in der Öffentlichkeit nachdrücklich vor einem Nachleben des Nationalsozialismus zu warnen. Er erklärt: „Ich betrachte das Nachleben des Nationalsozialismus in der Demokratie als potentiell bedrohlicher denn das Nachleben faschistischer Tendenzen gegen die Demokratie" (Adorno, 1959/1997, 555 f.). Zugleich äußert er die Befürchtung, dass die parlamentarische Demokratie von den Bürgern als eine Manifestation von Macht wahrgenommen würde. Dies wertet Adorno als ein Zeichen dafür, dass Demokratie ,nicht derart sich eingebürgert (hat), dass sie die Menschen wirklich als ihre eigene Sache erfahren, sich selbst als Subjekte der politischen Prozesse wissen“" (ebd., 559).

So hat Adorno in der Rolle des Intellektuellen in öffentlichen Vorträgen, in zahlreichen Rundfunkdiskussionen eindringlich darauf bestanden, dass die Demokratie in Deutschland nur eine Chance habe, wenn die Aufarbeitung der Vergangen- 
heit gelinge. Als in Deutschland der Vergangenheitsdiskurs durch den AuschwitzProzess in Frankfurt und den Eichmann-Prozess in Israel in der ersten Hälfte der sechziger Jahre noch höchst zögerlich, aber doch langsam anzulaufen beginnt, engagiert sich Adorno als Intellektueller, der an die Bürger einer politisch erstarkenden Öffentlichkeit appelliert. Diese intellektuelle Aufklärungsfunktion reflektiert er gesellschaftstheoretisch als „Wendung aufs Subjekt, Verstärkung von dessen Selbstbewusstsein" (ebd., 571). Er will mit rhetorischen Mitteln, mit provokativen Formulierungen die Öffentlichkeit aufrütteln; sie soll sich der Faktizität von Auschwitz stellen, dem, wofür der Name steht. Mit diesem Imperativ, den er nicht müde wird, gegen das Schweigen vorzutragen, nimmt Adorno eine agonale Position in der Öffentlichkeit ein: Er positioniert sich als militanter Neinsager, ja als Ruhestörer (Kramer, 1996, 513). Mit seiner Kritik an den verschiedenen Formen der Abwehr, die Schuld der Deutschen als Täter zum Gegenstand einer bewussten Auseinandersetzung zu machen, hatte er wesentlichen Anteil daran, dass damals ein Selbstverständigungsprozess über das Geschehene und über die Funktion der Demokratie in Gang kam. Er trägt nicht nur dazu bei, dass der normative Gehalt der demokratischen Verfassung überhaupt öffentlich thematisiert wird, sondern er ist einer jener Protagonisten, die für eine zweite, eine intellektuelle Gründung der Republik verantwortlich sind (Albrecht u.a., 1999; Bonacker, 1999, 170 ff.). So wurde Adorno durch das, was er selbst „Eingriffe“ nannte, zu einem Impulsgeber für die Prozesse der öffentlichen Meinungsbildung.

Freilich kontrastiert Adornos unermüdliche Kritik an der Schuldabwehr der Deutschen und ihrem mangelnden demokratischen Bewusstsein in auffälliger Weise mit seiner eigenen Haltung zum politischen Engagement. Adorno, orientiert an der exklusiven Kultur des bildungsbürgerlichen Kanons, nahm kein Blatt vor den Mund: „In Deutschland läuft vielfach das Engagement auf Geblök hinaus, auf das, was alle sagen, oder wenigstens latent gern hören möchten“ (Adorno, 1962/1997, 429). Er betont, dass es keineswegs die Aufgabe des Intellektuellen sein könne, sich für eine politische Programmatik, sei sie noch so fortschrittlich, einzusetzen. Denn diesem Einsatz ,zuliebe wird die politische Realität zu leicht gewogen: das mindert auch die politische Wirkung" (ebd., 418). Auch wenn Adorno sich gerade nicht zu den Stillen im Lande rechnen mochte, bei selbstgenügsamer Kontemplation nicht stehen bleiben wollte, ${ }^{4}$ so betrachtet er doch mit großer Skepsis die politisch motivierte Absicht jener Philosophen und Künstler wie beispielsweise Sartre und Brecht, die sich im Dienste des Fortschritts oder der revolutionären Sache in der Rolle des Intellektuellen mutig dünken, wenn sie ,aus Verzweiflung an der Gewalt kurzschlüssig zu der gewaltsamen Praxis überlauf(en)“" (Adorno, 1962/1997, 421). Für Adorno sollten Philosophie und Kunst nicht dafür benutzt werden, zur Tat aufzurufen oder Alternativen zu pointieren, „sondern durch nichts anderes als ihre Gestalt, dem Weltlauf widerstehen, der den Menschen immerzu die Pistole auf die Brust setzt" (ebd., 413). Analog versteht Adorno seine Rolle als Intellektueller nicht als Engagement für aufklärerische Politik, sondern als radikale Negation des Bestehenden. Adressiert ist sie nicht an die

4 Das steht keineswegs in Widerspruch damit, dass Adornos Philosophie in ihrem Karn cinc Philosophie der Kontemplation ist. Vgl. Martin Seel (2004), Adornos Philosophie der Kontemplation, Frankfurt am Main: Suhrkamp. 
autonome Öffentlichkeit, sondern an die Gesellschaft als einheitliches Subjekt. So wie er sich selbst als Außenseiter wahrnimmt, will er sich nicht aus der Perspektive des Teilnehmers am Diskurs der Öffentlichkeit beteiligen, sondern vom exterritorialen Ort aus. Sein Engagement als Intellektueller speist sich aus der „Kraft zur Dissidenz" (Jacques Derrida), der strikten Aufrechterhaltung von Distanz zum politischen Betrieb und gerade auch zu den vorherrschenden Tendenzen öffentlicher Meinungen, deren Ansprüche am Kriterium objektiver Wahrheit zu überprüfen sind.

Weil Adomo davon überzeugt ist, dass das äußerste Unheil zu Ende zu denken sei, repräsentiert Adorno die Gestalt des Intellektuellen als Dissident der Öffentlichkeit. Er isı in der deutschen Öffentlichkeit der Nachkriegsjahre, die er durch seine Interventionen in Gang brachte wie kaum ein anderer, gleichsam der Meinungsfremde. Adorno, für den Kommunikation immer schon halber Verrat an der Sache ist, verkörpert als Intellektueller bis in die Sprache hinein den antikonsensuellen Funktionstypus. Der Impuls des Nein-Sagens erstreckt sich generell auf das praktische Leben in der gegebenen Gesellschaft. Entsprechend hat Adomo postuliert: „Das Ziel richtiger Praxis wäre ihre eigene Abschaffung“ (Adorno, 1969a/1997. 769). Die Widersprüchlichkeit, in die er sich so manövriert, ist offenkundig. Auf der einen Seite plädiert er vehement für die Aufarbeitung der Vergangenheit und die Wahrnehmung der Chancen, die die demokratische Verfassung bietet. Auf der anderen Seite ist er der Überzeugung, dass die Sphäre der Politik ebenso Fassade ist wie die politische Öffentlichkeit, deren Medien er sich gleichwohl extensiv bedient. Adorno schlussfolgert denn auch in den Minima Moralia: „Wie der Intellektuelle es macht, macht er es falsch“" (Adomo, 1951/1997, 151).

\section{Habermas: Der konsensuelle Funktionstypus des Intellektuellen}

Kaum anders als Adorno repräsentiert Jürgen Habermas eine bestimmte intellektuelle Praxis. Während er sich früh schon und immer wieder mit dem Verhältnis von Theorie und Praxis beschäftigt hat, finden sich in seinem Werk nur am Rande Überlegungen zur Rolle des Intellektuellen in der Moderne. Alleine in einem Essay über Heinrich Heine von 1986 macht Habermas den Intellektuellen explizit zum Gegenstand der Reflexion. Diese Zurückhaltung bezüglich einer theoretischen Bestimmung des Intellektuellen ist umso auffälliger, als Habermas als der tonangebende Intellektuelle der Bonner und der Berliner Republik gelten kann; seine Reputation als streitbarer politischer Geist hat seit der gemeinsamen Initiative vom Mai 2003, bei der er die Federführung hatte, geradezu globale Ausmaße angenommen. Dieses in führenden Tageszeitungen gleichzeitig veröffentlichtes Plädoyer für ein eigenständiges Europa gegenüber einem hegemonialen Amerika, für eine Weltbürgergesellschaft auf der Grundlage kooperativer multilateraler Institutionen macht gleich auf den ersten Zeilen das zentrale Selbstverständnis der intellektuellen Praxis von Habermas deutlich: Er versteht sie als diskursiven Beitrag, der an die resonanzfähige Öffentlichkeit einer deliberativen Demokratie adressiert ist.

Die Funktion des Intellektuellen, der „sich mit rhetorisch zugespitzten Argumenten für verletzte Rechte und unterdrückte Wahrheiten, für fällige Neuerungen 
und verzögerte Fortschritte" (Habermas, 1987, 29) einsetzt, ist für Habermas historisch an die Konstitution einer politisch funktionsfähigen Öffentlichkeit gebunden, Öffentlichkeit verstanden als ,Medium und Verstärker einer demokratischen Willensbildung. Hier findet der Intellektuelle seinen Platz" (ebd., 28). Wenn der Intellektuelle sich innerhalb der öffentlichen Sphäre zu Wort meldet, verlässt er die Berufsrolle, wenngleich es für den praktischen Philosophen und kritischen Gesellschaftstheoretiker, der von der Wahrheitsfähigkeit praktischer Fragen ausgeht, Affinitäten zwischen seinen politischen Bewertungen und seinen theoretischen Grundannahmen gibt. So liegt es auf der Hand, dass eine Konsequenz der Kommunikationstheorie, die ja dem Nachweis gilt, dass in der alltagssprachlichen Kommunikation ein Telos gegenseitiger Verständigung eingebaut ist, darin besteht, die Produktivkraft Kommunikation praktisch unter Beweis zu stellen. Es ist in der Tat konkret zu demonstrieren, dass kommunikative Macht die politische Kultur zu bestimmen vermag und neben den Steuerungsmedien administrative Macht und Geld von Einfluss ist. Trotz dieser Wahlverwandtschaft zwischen dem Verständigungsparadigma und intellektueller Praxis ist für Habermas der Intellektuelle nichts weiter als ein aktiver Bürger, der sich im Nebenberuf neben anderen Aktivbürgern politisch engagiert, dies freilich ungefragt, ohne politisches Mandat tut. Sein Engagement ist von der „Verantwortlichkeit für das Ganze" (ebd., 52) getragen, nicht vom Ehrgeiz, selbst politische Macht zu erringen. Der Intellektuelle will nicht auf den politischen Machtkampf strategisch Einfluss nehmen, sondern kommunikativ, d. h. verständigungsorientiert auf die autonome und plurale Öffentlichkeit. Den Status des Intellektuellen gewinnt der Bürger nicht als geistige Autorität, nicht als professioneller Durchblicker, sondern als Diskursteilnehmer, der das besonders gut macht, was andere auch tun könnten: überzeugende Argumente liefern. Folglich resultiert die Anerkennung des Intellektuellen als Intellektueller aus der Qualität seiner kontroversen Argumente, die sich im Pro und Contra ihrerseits als Impulse für öffentliche Auseinandersetzungen bewähren müssen. Dabei drängen Intellektuelle keine Interpretationen auf, vielmehr sollen stets „die Adressaten (...) die unzweideutige Chance haben, angebotene Interpretationen unter geeigneten Umständen, d.h. zwanglos anzuerkennen oder abzuweisen. Aufklärung, die nicht in Einsicht, d.h. in zwanglos akzeptierten Deutungen terminiert, ist keine" (Habermas, 1981, 327).

Mit dieser Funktion praktischer Aufklärung hängt zusammen, dass der Intellektuelle Statthalter jener universalistischen Prinzipien ist, die den normativen Gehalt der Moderne, bzw. den Gehalt demokratisch verfasster Gesellschaften ausmachen. Diese Prinzipien verdanken sich der historischen Bewährung und Durchsetzung des „Projekts der Selbstermächtigung einer Gemeinschaft von Freien und Gleichen“ (Habermas, 1992, 467). Diese theoretische Funktionsbestimmung des Intellektuellen steht ganz im Einklang mit der intellektuellen Praxis von Habermas, der die Mentalitätsgeschichte der letzten Jahrzehnte durch eine ganze Reihe von Interventionen in Form von Artikeln, publizistischen Stellungnahmen, offenen Briefen, Diskussionen mit politischen Machtträgern geprägt hat:

- Sei es Mitte der sechziger Jahre seine Insistenz auf einer radikalen Hochschulreform und die Kontroverse mit dem Aktionismus der Studentenbewegung; 
- $\quad$ sei es der Historikerstreit, als Mitte der achtziger Jahre Geschichtswissenschaftler die Einmaligkeit der Naziverbrechen in Frage stellten;

- $\quad$ sei es die Debatte über zivilen Ungehorsam als kalkulierte Regelverletzung und über Verfassungspatriotismus, der an Stelle von Nationalität und Schicksalsgemeinschaft treten soll;

- $\quad$ sei es die Kritik am Golfkrieg und seiner Medieninszenierung;

- $\quad$ sei es seine Einmischung in der Frage Beitritt oder Anschluss der DDR nach dem Fall der Berliner Mauer;

- sei es die Äußerung zur Krise im Kosovo und zur militärischen Intervention, um Völkermord zu verhindern;

- sei es das aus der Katastrophengeschichte des letzten Jahrhunderts moralisch wie rechtlich gebotene Projekt der Verankerung universeller Menschenrechte in weltbürgerlicher Perspektive;

- sei es die energisch vorangetriebene Debatte über ethische Fragen der Reproduktionsmedizin und das Klonen;

- sei es die jüngste Stellungnahme zum völkerrechtswidrigen Vorgehen im zweiten Irak-Krieg seitens der USA-Regierung.

Was charakterisiert diese exemplarisch genannten Formen intellektueller Praxis? Vier Merkmale (Normativität, Diskursivität, Veränderbarkeit, Fallibilität) lassen sich u.E. herausstellen.

Erstens erstreckt sich der Gegenstandsbereich intellektueller Praxis auf alles, was Fragen des gerechten (nicht des guten) Lebens betrifft, die in den Entscheidungsbereich aller Menschen gehören. In der Rolle des Intellektuellen bringt Habermas eine moralische und damit implizit politische Haltung zum Ausdruck, der ein ausgeprägter Gerechtigkeitssinn zugrunde liegt: Gegebene Praktiken in Politik, Kultur und Gesellschaft werden im Lichte normativ ausgewiesener und begründeter Urteile zum Gegenstand der Auseinandersetzung gemacht. Dabei steht die Bezugnahme auf die universalistischen Verfassungsprinzipien der Demokratie, auf ihr republikanisches, verfassungspatriotisches Selbstverständnis im Vordergrund.

Zweitens beruht das Infragestellen darauf, zwischen richtigen und falschen Argumenten zu differenzieren, gute Gründe diskursiv vorzutragen, die auf zurechnungsfahige Rezipienten abzielen, die an die Sensibilität von Teilnehmern der öffentlichen Meinungs- und Willensbildungsprozesse appellativ gerichtet sind. Sinn und Zweck des öffentlichen Streits, den Habermas vom Zaun bricht, besteht darin, Diskursivität herzustellen; sagen wir: ein Modell für den öffentlichen Gebrauch der Vernunft vorzuführen.

Drittens ist den Interpretationen, die stets danach fragen, was im gemeinsamen Interesse aller liegt, der Impuls zumindest langfristiger praktischer Veränderung eigen, die Erwartung nämlich, dass bestehende Machtstrukturen, deren Illegalität aufgezeigt wird. auch gebrochen werden können.

viertens suggeriert Habermas keineswegs, dass sein intellektuelles Engagement privilegierten Einsichten in das zu verdanken ist, was insgeheim das gesellschaftliche Getriebe zusammenhält. Vielmehr setzt sich die intellektuelle Praxis, wie er selbst festgestellt hat, im besonderen Maße einem Irrtumsrisiko aus. Mit dieser Auf- 
fassung ist ein Verständnis des Intellektuellen unvereinbar. wonach er sich als Sinnvermittler und Deuter des Weltganzen stilisiert. „Der Denker als Lebensform, als Vision, als expressive Selbstdarstellung, das geht nicht mehr" (Habermas, 1985, 207).

So bleibt die intellektuelle Praxis überhaupt auf eine Übersetzungsfunktion beschränkt: auf die Übersetzung der komplexen Sachprobleme in den spezifischen Wertsphären der Wissenschaft, des Rechts, der Kunst in die Alltagssprache. In dieser Vermittlungsfunktion, gleichsam mehrere Sprachen sprechen, sich das Wissen verschiedener Expertenkulturen zu eigen machen zu können, ohne selbst Experte sein zu wollen, genau darin kulminiert die intellektuelle Kompetenz; sie besteht in Habermas eigenen Worten in ,eine( $r$ ) illuminierende( $n$ ) Förderung der totalitätsbezogenen Selbstverständigungsprozesse einer Lebenswelt" (Habermas, 1988, 26).

\section{Die Produktivkraft der Negation und der Kommunikation}

Für Adorno wie für Habermas ist die Katastrophengeschichte des Jahrhunderts der Extreme der provokativ wirkende Hintergrund ihrer Philosophie und Gesellschaftstheorie. Diese Gemeinsamkeit sollte nicht darüber hinwegtäuschen, dass beide einer anderen Generation angehören. Sie trennt die für das Denken des Älteren konstituive Erfahrung der Verfolgung durch den totalitären Staat, des Exils, des Verlusts der eigenen Kultur und Sprache in der Fremde. Für die lebensgeschichtliche Weichenstellung von Habermas war die Ablösung eines kriminellen Gesellschaftssystems durch die demokratische Verfassung entscheidend. Vielleicht ist heuristisch gesehen folgende Kontrastierung erlaubt: Während Adorno der Philosoph des Holocaust war, ist die Philosophie von Habermas eine nach dem Holocaust.

Es ist nicht zuletzt diese zeitgeschichtliche Situierung zum Zivilisationsbruch, die die jeweilige distinkte Art praktischer Kritik determiniert, die in den differenten Formen intellektueller Praxis zur Anwendung kommt. Für Adorno ist Kritik das Ganze seines Denkens: bestimmte Negation des Bestehenden im Medium des sprachlichen Ausdrucks. Kritik operiert im Modus bewusster Übertreibung, von der die Dialektik als letztem Mittel Gebrauch macht, um der Totalität des Verblendungszusammenhangs gewachsen zu sein. Kritik ist ein Verhalten, nämlich „Widerstand gegen (...) alles bloß Gesetzte, das mit dem Dasein sich rechtfertigt" (Adorno, 1969b/1997, 785). Die Erlösung bzw. Aufhebung der Verhältnisse durch Widerstand gegen das Ganze, auf die Kritik hofft, bezieht sich sowohl auf die Destruktivität des Zivilisationsprozesses, den drohenden Untergang der menschlichen Gattung als auch auf die utopischen Gehalte eines Richtigen Lebens, für die bei Adorno der Begriff des Nichtidentischen steht.

Demgegenüber ist für Habermas Kritik eine Beziehung zur sozialen Praxis, die im Lichte von moralischen Grundsätzen und Normen beurteilt wird, deren Rationalität selbst wieder kritisch zu hinterfragen ist. Kritik setzt konkret bei den gesellschaftlichen Institutionen an und bringt das Ausmaß der in sie eingelassenen strukturellen Gewalt zum Vorschein. Während Adorno aus einer exterritorialen Perspektive die zum System des Grauens gewordene Gesellschaft negiert, bezieht sich Habermas 
aus der Teilnehmerperspektive auf illegitime Formen politischer Macht in der Gesellschaft, er opponiert gegen jede Form des Dezisionismus. Adorno vertritt in seiner transzendierenden Gesellschaftskritik gleichsam die Partei des Auslandes. Hingegen kritisiert Habermas die Gesellschaft von der Partei des Inlandes her. Seine Kritik verdankt sich. so könnte man im Anschluss an eine Formulierung von ihm selbst sagen. der ..Kraft der Transzendenz von innen“". Oder, um Habermas nochmals zu Wort kommen zu lassen: Kritische Interventionen sind ,Inseln im Meer der Praxis" (Habermas. 1995. 23).

Um die Differenzen zwischen diesen beiden Typen von Kritik, die ihrerseits distinkte Typen intellektueller Praxis bedingen, etwas schärfer zu pointieren, möchten wir uns - mit einer gewissen Bedeutungsverschiebung - eine Unterscheidung zu eigen machen, die Habermas in einem Essay über Walter Benjamin eingeführt hat. Er kontrastiert den Typus einer rettenden Kritik mit dem einer bewusstmachenden Kritik (Habermas. 1998, 336 ff.). Mit diesem Terminus der rettenden Kritik lassen sich nicht nur die Intentionen von Benjamins Philosophie, sondern durchaus auch Eigentümlichkeiten von Adornos Selbstverständnis als Intellektueller charakterisieren. Natürlich ist Adomos Kritik eine argumentative und er hatte im Kontext historischer Erfahrungen gute Gründe für die Maxime, wonach das Ganze das Unwahre ist. Seine Kritik als bestimmte Negation resultiert in der Tat aus der realen Verzweiflung über den katastrophischen Geschichtsverlauf, als deren Opfer er sich sah. Aus diesem Grund ist seine Kritik als öffentlicher Intellektueller, die gerade nicht politisches Engagement sein wollte, vom Impuls der Rettung bzw. dem der Erlösung getragen. Diesen Gestus des Alles oder Nichts hat Adorno durch ein von ihm immer wieder herangezogenes Grabbe-Zitat veranschaulicht: „Denn nichts als nur Verzweiflung kann uns retten".

Bewusstmachende Kritik mag zwar aus Verzweiflung gespeist sein, aber sie ist nicht Ausdruck prinzipieller Resignation. Sie vertraut vielmehr auf die Veränderbarkeit sozialer Strukturen und auf das emanzipatorische Potenzial demokratischer Institutionen sowie auf die Einsichtsfähigkeit verständigungsorientiert handelnder Akteure. An die Handlungsakteure ist die bewusstmachende Kritik ja adressiert. Sie ist nicht vom subjektiven Impuls der Verzweiflung, sondern von dem der Entrüstung getragen. Sie ist der motivationale Grund, um Verfehlungen dingfest zu machen, indem aus der dialogischen Deutungsperspektive heraus, in der ersten Person Plural (wir), d.h. in der Form der begründeten Rede die Bedingungen und Folgen jener Verfehlungen nachgewiesen werden. Fluchtpunkt der Entrüstung über die Verletzung von Solidarität und Gerechtigkeit ist das diskursiv erzielte Einverständnis. So ist die bewusstmachende Kritik nicht objektiv besseres Wissen, sie ist nicht von einem externen Ausgangspunkt formuliert, sondern geht von der Binnenperspektive der eigenen Kultur aus. Sie setzt an den internen Strukturen systematisch verzerrter Kommunikation an. Sie diagnostiziert die inneren Konflikte und Widersprüche vor dem Hintergrund nicht nur faktisch geltender, sondern als gültig ausgewiesener bzw. auszuweisender Normen. Für die bewusstmachende Kritik gibt es keine letzten Antworten, weil dieser Typus intellektueller Praxis ein offener, fehlbarer, stets aufs Neue durchzuführender Prozess des Argumentierens ist. Die bewusstmachende Kritik vertraut weder den substantiellen Zielbestimmungen einer konkreten Utopie 
noch der Evidenz plötzlicher Einsichten, die in den Zustand einer versöhnten Menschheit münden. Bewusstmachende Kritik setzt auf die kontextsprengende Kraft universaler Geltungsansprüche und die Prozesseigenschaften, bei der öffentlichen Suche nach überzeugenden Argumenten.

\section{Ein dritter Typus intellektueller Praxis oder: „The guy in the ballcap with no education" - Selbstinszenierung und soziale Stilistik des Anti- Intellektuellen Michael Moore}

Wenden wir uns nun einem dritten Typus intellektueller Praxis zu, einem Mann nämlich, der gegenwärtig als ein Medienintellektueller besonderer Art gilt: Michael Moore, der weltweit erfolgreichste Dokumentarfilmer. An seinem Beispiel gilt es zu fragen, wie es ihm gelingt, sich in der gegenwärtigen Mediengesellschaft überhaupt Gehör zu verschaffen, ohne seine intellektuelle Autonomie zu verlieren, und von welchem Standpunkt aus er seine kritische Funktion innerhalb des öffentlichen Diskurses definiert.

Der Nicht-Journalist ist der erste, der investigativen Journalismus zu einem überwältigendem kommerziellen Erfolg gemacht hat, indem er detailreiche und furchtlose Recherchen mit humoristischen Darstellungsformen und publikumswirksamen Aktionen verbunden hat. Die Gestaltung und der Erfolg seiner filmischen und literarischen Produktion sind untrennbar mit seiner Person verbunden. Stets ist er als Autor und Akteur präsent, sein Bild, seine Handlungen und seine Stellungnahmen bilden den roten Faden der medialen Produktion. Im Folgenden stellen wir eine Analyse von Michael Moores medialer Selbstinszenierung an, die hier aus Platzgründen nur in Ausschnitten präsentiert wird.

Sie stützt sich auf seine Filme „Bowling for Columbine“ und „Fahrenheit 9/11", seine Auftritte bei verschiedenen Pressekonferenzen, bei der Oscar-Verleihung 2003, in Interviews und auf seiner Homepage. Die angeführten Zitate stammen jeweils von ihm selbst. ${ }^{5}$

Zentrale Konzepte unserer Analyse sind folgende:

- Soziale Stilistik: Welchen sozialen Stil der Selbstpräsentation und welchen sozialstilistisch markierten Habitus in Sprache, Kleidung und Verhalten präsentiert Michael Moore?

- Selbstpositionierung und Fremdpositionierung: Wie positioniert sich Moore selbst in der Kommunikation mit Publikum, Presse und Interviewpartnern? Wie positioniert Moore seine Adressaten, welche Einstellungen und Eigenschaften schreibt er ihnen zu?

5 Filme von Michael Moore: Bowling for Columbine, Regie: Michael Moore, 2003, DVD, 114 Minuten, zusätzlich: Special features: Interviews mit Michael Moore, Pressekonferenz mit Michasl Moore, Statements zum Film u.a.; Fahrenheit 9/II, Regie: Michael Moore, 2004, DVD, 122 Minuten, zusätzlich: Bonusmaterial 90 Minuten; Michael Moore in feuilletonistischen Besprechungen - z.B.: Die Feldzüge des Proleten, von Mike Davis, DIE ZEIT, Nr. 31. 22. Juli 2004, S. 37; Michael Moore. Manipulator, von Ludger Lüdgehaus, DIE ZEIT, 5. August 2004; Unrasicrte Störungen, von Josef Lederle. Rheinischer Merkur, Nr. 29/2004, S. 22 
- Sprachlich-kommunikative und rhetorische Verfahren: Welche sprachlichkommunikativen Verfahren benutzt Moore in unterschiedlichen kommunikativen Situationen? Welche rhetorischen und dialogsteuernden Techniken setzt er ein?

- Ideologie und Moral: Welche moralischen und ideologischen Positionen nimmt Moore ein? Wie stützt er sie. mit welchen Mitteln greift er die gegnerischen Positionen an?

\section{Soziale Stilistik und Selbstpräsentation}

Moore tritt nahezu immer (Ausnahme: Oscar-Verleihung) im gleichen körperlichen Habitus auf. der zu seinem visuellen Markenzeichen geworden ist: Turnschuhe, (ausgewaschenes) Sweatshirt, weite Jeans, kurzer Blouson oder alte schwarze Lederjacke, Baseballkappe, er ist erkennbar übergewichtig, unrasiert, trägt einen "formlosen" Vollbart und eine Hornbrille (,Kassengestell"), seine Haare wirken unfrisiert. Dieser Habitus entspricht dem eines Angehörigen der amerikanischen Arbeiterklasse (blue collar worker), er ist dezidiert unmodisch und nicht auf ästhetische Gestaltung und Wirkung orientiert. Dem korrespondiert auch seine Körperhaltung: er ist meist leicht geduckt, zieht den Kopf ein, die Schultern sind hochgezogen und nach innen gedreht. er geht mit den Händen in den Hosentaschen und zeigt die typische Haltung einer Wirbelsäulenskoliose und bemüht sich ganz offensichtlich nicht um ein körperlich attraktives, sportliches, elegantes etc. Erscheinungsbild. So zieht er auch in der Öffentlichkeit die Nase hoch oder kratzt sich. Moore erscheint als der kleine Mann von der Straße, der Nachbar, der neben dem Zuschauer in seinem Wohnvientel an der Supermarktkasse stehen könnte. Moore definiert sich bereits rein äußerlich als Mitglied der schweigenden Masse Amerikas, die in den Medien allein schon visuell nicht präsent ist: Moores Habitus ist in den Medien eine absolute Ausnahmeerscheinung. Kein professioneller Medienakteur tritt vergleichsweise unstilisiert, alltäglich und im Habitus der unteren Mittelklasse auf. Moores Habitus fehlt jede Stilisierung von Zugehörigkeit zu einer Bildungselite oder zu einer prestigereichen sozialen Schicht, er verzichtet aber ebenso auf Stilisierungen des Mondänen oder Ausgeflippten wie auf einen Künstlerhabitus (schwarzer Rollkragenpullover und schwarze Anzüge etc.), der angesichts seiner professionellen Identität (Dokumentarfilmer) zu erwarten wäre. Diese Darstellung von Nicht-Stilisjertheit verkörpert eine root-authenticity der sozialen Herkunft, die (gemäß einer weit verbreiteten Volksideologie) Ehrlichkeit und moralische Glaubwürdigkeit impliziert - Werte, die Moore immer wieder als Zentralwert und Richtschnur seines Schaffens in den Vordergrund stellt.

Moores Sprache ist in syntaktischer und lexikalischer Hinsicht eher einfach: Er benutzt kolloquiales Amerikanisch mit einigen familialen Wendungen, die er vor allem bew'ertend (z.B. bei pejorativen Personenkategorisierungen: ,jerks", „dude") und zur formelhaft-appellativen Zuspitzung (,don't get me this shit", „,your time is up") einsetzt. Gerade die familialen Wendungen werden häufig in ironischen, zitativen oder selbst-ironisch kommentierenden Kontexten verwendet. Moore macht 
meist kurze Sätze. Maximal bestehen sie aus einem Haupt- mit einem Nebensatz, so in argumentativen Kontexten oder zur Spezifikation ('x because $y^{\prime}$, ' $\mathrm{x}$ though $\mathrm{y}$ ', ' $\mathrm{x}$ where/when $\left.y^{\prime}\right)$. Seine Ausdrucksweise ist stets alltagsweltlich und leicht verständlich, allerdings meist sehr klar, ohne die für die gesprochene Alltagssprache typischen Selbstkorrekturen, Abbrüche oder Abtönungen. Fachsprachliche oder sozialstilistisch als akademisch, wissenschaftlich, bürokratisch oder einer spezifischen politischen Richtung zugehörig markierte Terminologie vermeidet er. Auch seine diskursiven Strategien sind weitgehend und in deutlicher Abweichung von sonstigen massenmedialen Gepflogenheiten diejenigen der informellen Allagskommunikation, allerdings in rhetorisch elaborierter Form (s.u.). Diese Elaboration wird jedoch erst unter dem analytischen Blick deutlich, in alltagsweltlicher Rezeptionseinstellung wirkt Moores Sprache und Kommunikationsverhalten wie das eines unstilisierten average American. In sprachlicher Hinsicht präsentiert sich also Moore als Stimme des Volkes, die auch komplexe Sachverhalte und moralische Anliegen in einer einfachen, allgemeinverständlichen Sprache formuliert. Er ordnet sich sprachlich weder einem politischen Standpunkt zu noch spricht er aus einer Position der expertischen Autorität (z.B. als Wissenschaftler, Journalist).

\section{Selbstpositionierung und Fremdpositionierung}

Moore stellt sich als Stimme der moralischen Mehrheit der Amerikaner dar: „I'm not a minority. I represent a majority of Americans, (...) but we just don't have a voice. We don't own the media (...).“ Er legt größten Wert darauf, nicht als Vertreter einer politischen Minderheiten-Position abgetan zu werden: „There are millions and millions and millions of people who are saying the same things as I say. I'm not alone in this." Dabei reklamiert er für seine journalistischen Erkenntnisse, seine Ansichten und Aktionen keinerlei Originalität, Exklusivität oder besondere Anerkennung. Er stellt sich als Person dar, die lediglich artikuliert, was die meisten denken und erfahren haben, und die diejenigen Aktionen unternimmt, Fragen stellt und Dinge untersucht, die alle schon immer einmal wissen bzw. getan sehen wollten. Sehr häufig formuliert er seine Positionen in der ersten Person Plural (we), und er nutzt jede Gelegenheit, um Kollektividentitäten zu formieren und zu präsentieren und um kollektive Solidaritäten zu konstituieren (z.B. Entgegennahme des Oscars gemeinsam mit dem Filmteam, häufige Benutzung von Publikum-inkludierendem we (., do they think we're stupid?", Solidarisierung mit Opfern in seinen Filmen, Aktionen für Opfer und mit ihnen (z.B. K-Mart-Episode in „Bowling for Columbine“)). Gemeinschaft und Repräsentanz werden dabei von ihm oft kontrafaktisch unterstellt, Erfolge werden ebenso kontrafaktisch den von ihm vertretenen Gruppen zugeschrieben, nicht von ihm für sich selbst reklamiert. Moore gibt sich bescheiden. Er tritt als uneigennütziger Anwalt und Tribun des Volkes auf, der dieses versteht und repräsentiert, da er aus seiner Mitte kommt und ihm gleicht. Er vermeidet es, mit einer politischen Partei oder ideologischen Position identifiziert zu werden oder als jemand zu erscheinen, der seine Interessen aus einem theoretisch, ideologisch etc. voraussetzungsvollen Hintergrund gewinnt. Er stellt sich vielmehr als Agenten des common 
sense dar: Sein Gestus ist es, unbequeme Wahrheiten auszusprechen, die jeden vernünftigen Menschen angehen und evident sind. Moore vermeidet es, als marginalisierter oder besserwisserischer Intellektueller zu erscheinen. So verweist er auf sein abgebrochenes Studium und wirft den Journalisten vor, dass er als unbedarfter, einfacher Mann die Arbeit von Leuten mache ,who are much smarter than I am“.

In Übereinstimmung mit seinem Habitus betont er seine regionale und nationale Identität. Seine Herkunft aus dem im Niedergang befindlichen Industrieort Flint bildete schon den Ausgangspunkt für seinen ersten erfolgreichen Film „Roger \& Me“. Moore inszeniert sich als Sprachrohr der kleinen Leute von dort, kommt immer wieder auf die konkreten Verhältnisse in seinem Heimatort $\mathrm{zu}$ sprechen und hebt seine regionale und seine sozialstilistische Verwurzlung hervor („I am the guy in the ballcap with no education"). Gleichzeitig betont er seine Identität als Amerikaner, will Kritik dezidiert als patriotischen Dienst und nicht als Nestbeschmutzung und Anti-Amerikanismus verstanden wissen (,I'm an American and my job is to stay there and trying to fix things there"). Seine häufigen Kontrastierungen der von ihm angeprangerten Zustände in den USA mit anderen Staaten (v.a. Kanada) rahmt er als Ansporn zur Besserung. Er negiert, damit eine Kränkung des nationalen Selbstbewusstseins zu beabsichtigen, was allerdings bei der Systematik und Häufigkeit der negativen Vergleiche der USA mit dem Ausland sehr nahe liegt.

Moores Fremdpositionierung in seinen öffentlichen Auftritten ist sehr polarisierend. Er konstruiert eine klare, schwarz-weiße Dichotomie zwischen sich und dem von ihm mit ihm identifizierten Publikum einerseits und einem jeweiligen Gegner andererseits. Dieser wird polemisch und ohne Differenzierung unlauterer Motive, verbrecherischer Handlungen, negativer Einstellungen und Charakterzüge bezichtigt. Die interaktive Polarisierung wird durch direkte Adressierung des abwesenden Gegners (,Your time is up Mr Bush") dramatisiert, das Publikum wird performativ in einen symbolischen Kampf als Partei eingebunden. Indem sich Moore mit Vorliebe mit übermächtigen Gegnern (Konzernen, Politikern, Verbänden/Lobbies, staatl. Institutionen) anlegt, demonstriert er die Attitüde des furchtlosen Aufklärers, der tatkräftig und schnörkellos zur Sache schreitet. Charakteristisch ist dabei ein Gestus der Zuversicht und Siegesgewissheit, der auch nach offensichtlichen Niederlagen (z.B. Wiederwahl Bushs) unverdrossen beibehalten und durch relativierende Argumente untermauert wird. Die permanente Wiederholung der Prophezeiung eines Wandels zum Guten und die stetige Wiederholung des Aufrufs zum Engagement verleiht Moores Auftreten eine missionarisch-messianische Qualität, die aber niemals eine ideologisch-parteiliche Färbung einnimmt, sondern stets im Namen einer Einsicht, die eigentlich alle schon haben, auftritt.

In seinen Interviews behandelt er seine Gegner (Interviewpartner) mit Ernsthaftigkeit und Respekt. was die sachliche Auseinandersetzung betrifft. Die interaktive Etikette respektiert er dagegen häufig nicht (s.u.). 


\section{Sprachlich-kommunikative und rhetorische Verfahren}

Moores kommunikativer Stil unterscheidet sich sehr stark je nach Kommunikationstyp. In seinen investigativen Interviews agiert er zunächst nie polarisierend. Er verzichtet auf Unterstellungen und zeigt sich anfänglich empathisch gegenüber seinen Interviewpartnern, er stellt keine vorurteilsgeladenen oder präsuppositionshaltigen Fragen, sondern beginnt mit Informations- und Begründungsfragen, die oftmals auf die Biographie des Befragten und seine eigenen Erfahrungen abheben. Dem eingeweihten Zuschauer, der Moores Position kennt, erscheint er oft naiv oder scheinheilig, da er Positionen, Sachverhalte und Begründungen erfragt. die als bekannt vorausgesetzt werden können. Genau diese naiven Fragen sind aber notwendig, um Einschätzungen seiner Gegner als durch diese selbst bzw. durch von ihnen gelieferte Fakten fundiert erscheinen zu lassen. Interaktionsorganisatorisch sind sie die Voraussetzung dafür, dass seine Interviewpartner Material liefern, an denen Moore seine Entgegnungen ansetzen kann, da er sie nur so des Widerspruchs, der Inkonsequenz oder der Unfundiertheit ihrer Haltungen (für den Zuschauer) überführen kann. Anfänglich bestätigt er seine Interviewpartner (z.B. durch kooperative Ergänzungen, Zustimmungen oder Paraphrasen). Im Verlauf des Interviews insistiert er auch gegen das offensichtliche Unbehagen und den Widerstand seiner Interviewpartner und beginnt sie mit Vorwürfen, Kritik und unliebsamen Feststellungen zu konfrontieren. Tabus werden offen und direkt, aber ohne Markierung ihres tabuisierten Charakters angesprochen. Dabei ignoriert er hartnäckig die Themen- und Gesprächsbeendigungsinitiativen seiner Partner, bleibt aber stets sachlich, wird nicht persönlich verletzend, macht sich nicht über die Interviewpartner lustig, legt es nicht auf persönliche Bloßstellungen an und paktiert nicht mit dem Zuschauer gegen sie (z.B. durch nonverbale Kommentare zur Kamera). Er stellt ihnen keine Fallen, argumentiert aber ernsthaft und nachdrücklich und versucht sie in die Argumentation hineinzuziehen und $\mathrm{zu}$ argumentativen Zugeständnissen $\mathrm{zu}$ bewegen. Wesentliches Argumentationsverfahren für seine Position ist dabei Moores Rekurs auf moralische Standards, die der Adressat nicht ablehnen kann, ohne sich in Selbstwiderspruch zu begehen oder eine moralisch unhaltbare Position einzunehmen.

Sowohl in seinen Interviews wie auch in seinen öffentlichen Aktionen tritt Moore für das Publikum als koboldartig-subversiver Agent des kleinen Mannes auf: Er traut sich „einfache“ Dinge, die wirkungsvoll sind und jedem einleuchten, geht direkt zu den Verantwortlichen, stellt die Schuldigen und legt ihnen die Fragen vor, die sonst keiner auszusprechen wagt. Soziale Probleme werden von ihm exemplarisch konkretisiert, personalisiert und in den Bereich des direkten Handelns zurückgeholt, damit auch - gegen die Anonymität makrosozialer und politischer Prozesse potenziell für die Wirkungsreichweite des Einzelnen zurückgewonnen. Insofern ist Moores konkretistisches Vorgehen eine Form von vorexerziertem empowerment und eine Simplifizierung von komplexen Vorgängen auf elementare, moralisch und personal interpretierbare Wahrheiten. Er demonstriert ein pragmatisches, unmittelbar resultats- und lösungsorientiertes Verständnis von politischen und sozialen Problemen, und in aufklärerischer Hinsicht ein ebenso pragmatisches Verständnis der Orientierung an den Fakten selbst, die er aufsucht und zum Sprechen bringt. 
In seinen öffemlichen Reden tritt Moore als agitatorischer Volkstribun auf, der alle Register der populistischen Rhetorik zieht. So funktionalisiert er das Genre der Dankesrede bei der Oscar-Verleihung um zu einer politischen Propagandarede mit abschließender Moralisierung und prophezeiender Beschwörung ,We are against this war, Mr Bush. Shame on you! Your time is up." In seinen Redeauftritten befleiBigt er sich der actio der polemischen Rede an das Volk: er macht regelmäßige Taktgesten mit geballter Faust (abwechselnd links rechts), sein Blick geht von links nach rechts und zurück durchs Publikum, er benutzt viele illustrative Gesten (z.B. horizontale Schlussgeste zu „your time is up“), weisende (richtungs- und personaldeiktische) und Betgesten. Die Gestik ist generell kraftvoll und bestimmt. Moore betreibt die sy'stematische (Ein-)Prägung von Schlüsselwörtern und -phrasen, die er unablässig innerhalb einer und in mehreren Reden wiederholt. So z.B. in der OscarPreisrede das Wort „fictitious“, das er in gut einer Minute sieben Mal wiederholt: "we like non fiction and we live in fictitious times we live in a time where we have fictitious election results and that elects a fictitious president we live in a time where we have a man sending us to war for fictitious reasons whether it's the fictitious of duct tape or the fictition of orange alert". Auch in lexikalischer Hinsicht werden systematisch binäre Oppositionen aufgebaut, die den Positionen und Identitäten von we und they entsprechen, z.B. "non-fiction" vs. "fictitious", "solidarity" vs. "egoism". Seine Reden zeichnen sich durch sehr viele inhaltliche, teils auch wörtliche Wiederholungen aus. Prosodisch benutzt er häufig ein emphatisches Pathosmuster, das sich durch Rhythmisierung mit Vibrato, auffälligen Vokallängungen und singenden Intonationskonturen auszeichnet. Dieses Muster zeugt von Nachdruck und emotionaler Inbrunst. Moore spricht sehr laut bis schreiend, er fordert durch gezielte Pointensetzung und sloganhafte Zuspitzung Beifall heraus und schreit dann gegen den Beifall an. was eine weitere Anfeuerung des Publikums impliziert. Seine Themenbehandlung ist (im Gegensatz zu seinen Interviews) thetisch, unmodalisiert, unargumentativ (keine Begründungen) und nicht differenzierend. Abschwächungen, Gegenargumente. Ungewissheiten, Konditionale etc. fehlen. Moore arbeitet mit humoristischen Elementen wie Wortspielen (z.B. „embedded - in bed“: „I'm embedded in the army I'm in bed with the pentagon I'm in bed with the Bush administration", auch hier: Wiederholung und Steigerungsdynamik/Amplificatio, Pointendramaturgie, vom Flüstern zum Schreien, Schlüsselwort-Bildung) und Redewiedergaben, wobei er die (stilisierten) Stimmen gern als Karikaturverfahren einsetzt, so z.B. wenn er das Atombomben besitzende Nordkorea als ungehorsamen Schüler auftreten lässt, der vergeblich darum bittet, bestraft zu werden, oder einen ängstlichen Amerikaner inszeniert, der sich übertrieben vor Bin Laden fürchtet („don't kill me Osama“). Moore produziert hier Komik und Klamauk mit Unterhaltungswert, die der karikativen Veranschaulichung von Einstellungen dienen, die provokativ überzeichnet und persifliert werden. Zusammen mit dem Vollzug symbolischer Konfrontationshandlungen (z.B. Anruf im weißen Haus, in dem Moore erklärt, Bushs Zeit sei abgelaufen) inszeniert Moore so eine Anschaulichkeit und Unmittelbarkeit der Konfrontation und der Möglichkeit der direkten Intervention, die zur Erzeugung von Gemeinschaftserlebnissen der Selbstbestätigung eigener Macht und Rechtsbewusstseins und der Vergewisserung des gemeinsamen Feindes dienen. Gleichzeitig balanciert er so 
die Attitüde des propagandierenden Demagogen mit dem des humoristischen Schalks, der stets für einen Witz oder eine gelungene Parodie gut ist: Nie inszeniert Moore eine militante oder verbissene Form der Auseinandersetzung, sondern stets werden politisches Engagement und Konflikt mit Humor und Slapstick kombiniert.

In Presseinterviews und -konferenzen tritt Moore als ruhiger, argumentativer, schnörkellos antwortender, ironischer und zuweilen auch selbstironischer Dialogpartner auf. Er präferiert klare, einfache Argumentationen mit These-Begründungsstruktur, benutzt häufig eine dozierende Gestik, die Mimik ist entspannt. Seine Diktion ist einfach, insbesondere benutzt er alltagsweltliche Diskursstrategien, die in den Medien sonst kaum anzutreffen sind: Er erzählt ausführlich aus der eigenen Biographie, appelliert an Erfahrungen, die jeder kennt, benutzt Redewiedergaben, mit denen er die Haltungen verschiedener Beteiligter theatralisch inszeniert. So z.B. beim Kontrast der solidarischen Deutschen mit den egoistischen Amerikanern: „We're all on the same boat we're all German. We have to take care of each other. (...) We have a responsibility to see that this person gets a doctor.“" vs. „Every man for himself. Pull yourself up by your bootstraps. Me, me, me, me, me." Die Redewiedergaben werden mit entsprechender stimmlicher Imitation, Mimik und Gestik veranschaulicht, teilweise auch deutlich als humoristische Fiktionalisierungen mit Unterhaltungsintention gerahmt. Moore singt, er gebraucht evokative Bilder und Szenen, einfache Metaphern und argumentiert fast immer mit Beispielen und konkreten Fällen, nie nur theoretisch oder mit Statistiken. Moore stellt sich selbst als neugierigen, atheoretischen Menschen dar, der eine klare moralische Position hat, aber stets faktenorientiert und nicht vorurteilshaft ist. Er moralisiert, aber ohne Besserwisserei, und verzichtet auf jede intellektuelle oder arrogante Attitüde.

\section{Ideologie und Moral}

Wie schon dargestellt, ordnet sich Moore keiner dezidierten ideologischen Position $\mathrm{zu}$, und er verzichtet auf alle theoriegeladenen Argumentationen. Politische Bekenntnisse (für die Demokraten) werden von ihm zwar geäußert, immer aber mit dem einschränkenden Hinweis, es handele sich um die Wahl des geringeren Übels. Seine permanent propagierten Leitdifferenzen sind Lüge und Täuschung vs. Wahrheit' und 'Gerechtigkeit vs. Ungerechtigkeit'. Ehrlichkeit, Glaubwürdigkeit und Gerechtigkeit werden als absolute, nicht zu hinterfragende Werte, die jedes soziale Zusammenleben leiten sollen, propagiert. Diese Werte werden als leicht verständlich, selbstevident und aufgrund des gesunden Menschenverstandes in ihrer (Nicht-) Realisierung erkennbar dargestellt. Die Pole von 'gut' und 'böse' stellt er so dar, als seien sie im jeweiligen Falle zweifels- und problemlos zu bestimmen. Komplexitäten moralischer Positionen, kontingente Verwicklungen, moralische Indifferenzzonen gibt es bei ihm nicht. Dagegen fordert er für andere Themen Entmoralisierung (z.B. Schwulenehe). Moore kontrastiert den moralischen Charakter des amerikanischen Individuums mit der Unmoralität des Systems und der Mächtigen: „I want the goodness of us as individuals to connect to the goodness of us as a whole." Er vertritt eine Ethik der Anständigkeit des kleinen Mannes, die es als Maßstab für die 
große Politik zu etablieren gelte. Diese wird demgegenüber als korrupt, unmenschlich und aggressiv dargestellt. Moores Moralisierung wird immer wieder gebrochen durch humoristische und komödiantische Elemente, die überraschend, entspannend und unterhaltsam wirken. Der Dokumentarfilmer Michael Moore agiert wie ein allerdings freundlicher - Wolf im Schafspelz, dem es gelingt, nicht als Intellektueller, sondern vielmehr als Anti-Intellektueller die Abwehrlinien des politischen Establishments zu unterlaufen und zu hintergehen, um der Stimme des kleinen Mannes (the people) in der Öffentlichkeit Gehör zu verschaffen.

\section{Ausblick: Typen intellektueller Praxis}

Die Ausführungen gaben einen ersten Einblick in die Analyse von Wandlungstendenzen der Formen und Funktionen des Intellektuellen in der Gegenwart. Ausgangspunkt war die Rekonstruktion der typologischen Eigenart des Denkstils repräsentativer Intellektueller (Adomo und Habermas), die in öffentlichen Auseinandersetzungen das Wort ergriffen haben - kontrastiert mit einem aktuellen Beispiel öffentlicher Kritik, die pointiert auf die Strukturen und Dynamiken der Medienkommunikation setzt und sie auszureizen weiß (Michael Moore). Die skizzierte Untersuchungsperspektive soll in Folgeuntersuchungen erweitert und vertieft werden, insbesondere auch in Abgrenzung von den öffentlichen Stellungnahmen anderer sozialer Gruppierungen wie etwa Politiker, Journalisten, Experten. Die zentrale Forschungsfrage lautet, welche zeitgeschichtlichen Veränderungen der intellektuellen Kritikform in und fir die demokratische (Medien-)Öffentlichkeit festzustellen ist (MüllerDoohm, 2005; Neumann-Braun, 2005).

\section{Literatur}

Adorno, Theodor W. (1951/1997), Minima Moralia, Gesammelte Schriften, hrsg. von Rolf Tiedemann. Bd. 4, Frankfurt am Main: Suhrkamp.

Ders. (1959/1997), Was bedeutet Aufarbeitung der Vergangenheit, Gesammelte Schriften, hrsg. von Rolf Tiedemann, Bd. 10.2, Frankfurt am Main: Suhrkamp.

Ders. (1962/1997), Engagemem. Gesammelte Schriften, hrsg. von Rolf Tiedemann, Bd. 11, Frankfurt am Main: Suhrkamp.

Ders. (1969a/1997), Marginalien zu Theorie und Praxis, Gesammelte Schriften, hrsg. von Rolf Tiedemann, Bd. 10.2, Frankfurt am Main: Suhrkamp.

Ders. (1969b/1997). Sritik, Gesammelte Schriften, hrsg. von Rolf Tiedemann, Bd. 10.2, Frankfurt an Main: Suhrkamp.

Ders. und Thomas Mann (2002), Briefwechsel 1943-1955, hrsg. von Christopf Gödde / Thomas Sprecher. Frankfurt am Main: Suhrkamp.

Albrecht, Clemens: Günter C. Behrmann, Michael Bock, Harald Homann / Friedrich H. Tenbruck (1999). Die intelleknelle Grïndung der Bundesrepublik, Frankfurt/New York: Campus. 
Bonacker, Thorsten (1999) / Theodor W. Adorno - Die Zukunft des Erinnerns, in: Claudia Fröhlich / Michael Kohlstruck (Hg.): Engagierte Demokraten. Vergangenheitspolitik in kritischer Absicht Münster: Westfälisches Dampfboot.

Gilcher-Holtey, Ingrid (1997), Menschenrechte oder Vaterland? Die Formierung der Intellektuellen in der Affäre Dreyfus, Berliner Journal für Soziologie, Bd. 7.

Habermas, Jürgen (1981), Die Utopie des guten Herrschers, Kleine Politische Schriften I-IV, Frankfurt am Main: Suhrkamp.

Ders. (1985), Die Neue Unübersichtlichkeit, Frankfurt am Main: Suhrkamp.

Ders. (1987), Heinrich Heine und die Rolle des Intellektuellen in Deutschland, in: ders.: Eine Art Schadensabwicklung, Frankfurt am Main: Suhrkamp.

Ders. (1988), Nachmetaphysisches Denken, Frankfurt am Main: Suhrkamp.

Ders. (1992), Faktizität und Geltung, Frankfurt am Main: Suhrkamp.

Ders. (1995), Die Normalität einer Berliner Republik. Kleine Politische Schriften VIII, Frankfurt am Main: Suhrkamp.

Ders. (1998), Philosophisch-politische Profile, Frankfurt am Main: Suhrkamp.

Karadi, Éva / Erszébet Vezér / Georg Lukács (1985), Karl Mannheim und der Sonntagskreis, Frankfurt am Main: Sandler.

Kramer, Sven (1996), „Wahr sind die Sätze als Impuls ...” Begriffsarbeit und sprachliche Darstellung in Adornos Reflexionen auf Auschwitz, Deutsche Vierteljahresschrift fiir Literaturwissenschaft und Geistesgeschichte, 70. Jg., Heft 3.

Müller-Doohm, Stefan (2005) / Theodor W. Adorno / Jürgen Habermas - Two Ways of Being a Public Intellectual, in: European Journal of Social Theory 8 (4), London 2005 (im Druck).

Ders. (2003), Adorno. Eine Biographie, Frankfurt am Main: Suhrkamp.

Ders. (2005), Zur Soziologie intellektueller Kritik, Oldenburg (im Manuskript).

Neumann-Braun, Klaus (2005), Medienintellektuelle als Teil kritischer Öffentlichkeit, Landau (im Manuskript).

Oevermann, Ulrich (2001), Der Intellektuelle. Soziologische Strukturbestimmung des Komplementär von Öffentlichkeit, in: Andreas Franzmann / Sascha Liebermann / Jörg Tykwer, Hrsg., Die Macht des Geistes. Soziologische Fallanalysen zum Strukturtyp des Intellektuellen, Frankfurt am Main: Humanities Online.

Offe, Claus (2003), Herausforderungen der Demokratie, Frankfurt am Main/New York: Campus.

Taubes, Jacob (1996), Die Intellektuellen und die Universität, in: ders.: Vom Kult zur Kultur: Bausteine zu einer Kritik der historischen Vermunft, München: Fink. 